\title{
An integrated fingerprinting and kinetic approach to accelerated shelf-life testing of chemical changes in thermally treated carrot puree
}

Biniam T. Kebede**, Tara Grauwet, Johannes Magpusao, Stijn Palmers, Chris Michiels, Marc Hendrickx, Ann Van Loey*

All authors are affiliated to:

Centre for Food and Microbial Technology

Department of Microbial and Molecular Systems ( $\left.\mathrm{M}^{2} \mathrm{~S}\right)$

KU Leuven

Kasteelpark Arenberg 23 - box 2457

3001 Heverlee

Belgium

http://www.biw.kuleuven.be/m2s/clmt/lmt

*Author to whom correspondence should be addressed on post publication

Fax: +32-16-32.19.60

Telephone: $+32-16-32.15 .67$

E-mail: ann.vanloey@biw.kuleuven.be

**Author to whom correspondence should be addressed during submission process

Fax: +3216321960

Telephone: +3216376763

E-mail: BiniamTamiru.Kebede@biw.kuleuven.be 


\begin{abstract}
$\underline{\text { Abstract }}$
To have a better understanding on chemical reactions during shelf-life, an integrated analytical and engineering toolbox: "fingerprinting-kinetics" was used. As a case study, a thermally sterilized carrot puree was selected. Sterilized purees were stored at four storage temperatures as a function of time. Fingerprinting enabled selection of volatiles clearly changing during shelf-life. Only these volatiles were identified and further zoomed into. Next, kinetic modelling was performed to investigate the suitability of these volatiles as quality indices (markers) for accelerated shelf-life testing (ASLT). Fingerprinting enabled selection of terpenoids, phenylpropanoids, fatty acid derivatives, Strecker aldehydes and sulfurcompounds as volatiles clearly changing during shelf-life. The amount of Strecker aldehydes increased during storage, whereas the rest of the volatiles decreased. Out of the volatiles, based on the applied kinetic modelling, myristicin, $\alpha$-terpinolene, L- $\beta$-pinene, $\alpha$-terpineol and octanal were identified as potential markers for ASLT.
\end{abstract}

\title{
Keywords
}

Thermal sterilization; carrot puree; accelerated shelf-life testing; headspace GC-MS fingerprinting; kinetic modelling; chemical reactions.

\section{Abbreviated running title}

Fingerprinting-kinetics of shelf-life testing of sterilized carrot 


\section{INTRODUCTION}

2 Theoretically, when based on microbiological safety, sterilized food can be stored indefinitely.

3 However, the best before date is limited due to chemical changes whether or not triggered during

4 processing and incessantly taking place during shelf-life (van Boekel et al., 2010). Considering

5 consumer expectations, quality should be maintained at a targeted level during the period

6 between processing and purchase as well as between purchase and consumption. There is a need

7 for studies which not only take into account quality changes during processing, but also as a

8 function of shelf-life. For practical reasons, especially when the actual storage time is long,

9 shelf-life studies are based on accelerated shelf-life testing (ASLT) techniques that considerably

10 shorten the process of obtaining the necessary experimental data (Mizrahi, 2000). ASLT works

11 with a basic assumption that the effects of extrinsic parameters (mostly temperature) on the rate

12 of deteriorative reactions can be quantified by applying the principle of chemical kinetics

13 (Labuza \& Taoukis, 1990; Mizrahi, 2000; Robertson, 2000). In other words, with the use of

14 increased temperature as abuse condition and assuming that reactions follow Arrhenius kinetics,

15 deterioration rates at ambient/normal distribution conditions can be extrapolated from those of

16 elevated temperatures (Hough, Garitta, \& Gomez, 2006; Corradini \& Peleg, 2007).

18 Traditionally, quality investigations during shelf-life have been performed using an univariate

19 approach in which the change in food quality was tailored either with the loss of predetermined quantifiable quality indices such as nutrient or by the formation of an undesirable off-flavor

21 and/or discoloration of compounds (Sithole, McDaniel, \& Goddik, 2005). This strategy is the most straightforward way to address such quality investigations, and the corresponding results are undoubtedly of great value. However, fixating on known quality aspects entails that possible different effects are overlooked. Since food degradations are caused by the interaction of many attributes, more comprehensive results can be collected, that former methodologies were not 
capable of doing, for example using currently existing food chemometric tools (Saavedra, Cordova, Galvez, Quezada, \& Navarro, 2013).

In the present work, to have a better understanding on chemical reactions during shelf-life, the use of an advanced analytical method, relevant data preprocessing methods, multivariate statistical techniques and kinetic models were integrated to develop an analytical and engineering toolbox, called "fingerprinting-kinetics". As a case study, a thermally sterilized carrot puree was selected. Sterilized purees were stored at four different temperatures: $20^{\circ} \mathrm{C}, 28$ ${ }^{\circ} \mathrm{C}, 35^{\circ} \mathrm{C}$ and $42{ }^{\circ} \mathrm{C}$. In this work, quality changes linked to the volatile fraction were studied. Volatiles are often linked to process-induced reactions and have a major contribution to food flavor. Volatiles, being regularly degradation products of major food components (e.g., sugar, fat, nutrients), they can be approached as witnesses for what is happening in a complex food system. The volatile fraction of the samples was analyzed with a headspace solid-phase microextraction GC-MS (HS-SPME-GC-MS) fingerprinting procedure as a function of storage times and temperatures (kinetics). This approach considers all compounds detected in the investigated food fraction. Within a fingerprint procedure at the start of the analysis all the compounds are unknowns, it has been called an "untargeted approach" before (Grauwet, Vervoort, Colle, Van Loey, \& Hendrickx, 2014). The amount of data generated using a HSSPME-GC-MS analysis might be overwhelming. Multivariate statistical data analysis (MVDA) techniques are most appropriate for extracting important information out of these large data sets by reducing the dimensions of the multivariate data to a few manageable dimensions.

The objective of the present work was twofold. As a first objective, the potential of fingerprinting was used as fast screening technique for monitoring chemical changes during shelf-life. In other words, fingerprinting could enable selection of volatiles of which detected amounts are changing the most during shelf-life. Only these molecular entities were identified 
and further zoomed into (to link them to possible reaction pathways). The power of fingerprinting to identify important reaction pathways within a complex of chemical changes was clearly demonstrated in our previous studies (Vervoort et al., 2012; Kebede et al., 2013; Kebede et al., 2014a; Kebede et al., 2014b).

Since conducting shelf-life study at the normal storage temperature can be quite resource consuming, as a second objective of this work, the potential of ASLT was investigated. Based on the data from the fingerprinting as a function of storage times and temperatures, a kinetic modelling was performed to investigate the reaction kinetics of volatiles (selected by the fingerprinting approach) at different storage temperatures. By evaluating the estimated kinetic parameters, the suitability of these volatiles as quality indices (markers) for ASLT was investigated (Fig. 1).

\section{MATERIALS AND METHODS}

\subsection{Sample preparation}

A single batch of freshly harvested carrots (cv. Nerac) was purchased at a local market. The carrots were carefully washed, peeled and cut into standardized cylindrical pieces of approximately $1 \mathrm{~cm}$ thickness. The carrot cubes were packed into low-density polyethylene bags. To prevent enzymatic reactions during processing, storage and thawing, the packaged carrots were blanched at $95{ }^{\circ} \mathrm{C}$ for $8 \mathrm{~min}$ in a water bath (Haake W15 DC-10, Clausthal-Zellerfeld, Germany). The blanching conditions were validated using a qualitative and quantitative peroxidase test (Adebooye, Vijayalakshmi, \& Singh, 2008). After blanching, the plastic bags were immediately cooled in ice water for $10 \mathrm{~min}$, frozen in liquid nitrogen and stored in a freezer at $-40{ }^{\circ} \mathrm{C}$ until processing. Prior to processing, the samples were thawed overnight at $4{ }^{\circ} \mathrm{C}$. In order to prepare the puree, deionized water was added to the blanched carrot, blended for 1 min using a Buchi mixer (B-400, BUCHI, Switzerland) and further homogenized by high pressure 
homogenization (at 1000 bar while temperature maintained $<4{ }^{\circ} \mathrm{C}$ ) (Panda $2 \mathrm{~K}$, Gea Niro Soavi,

Mechelen, Belgium). The sample preparation steps are schematically listed in Fig. 1.

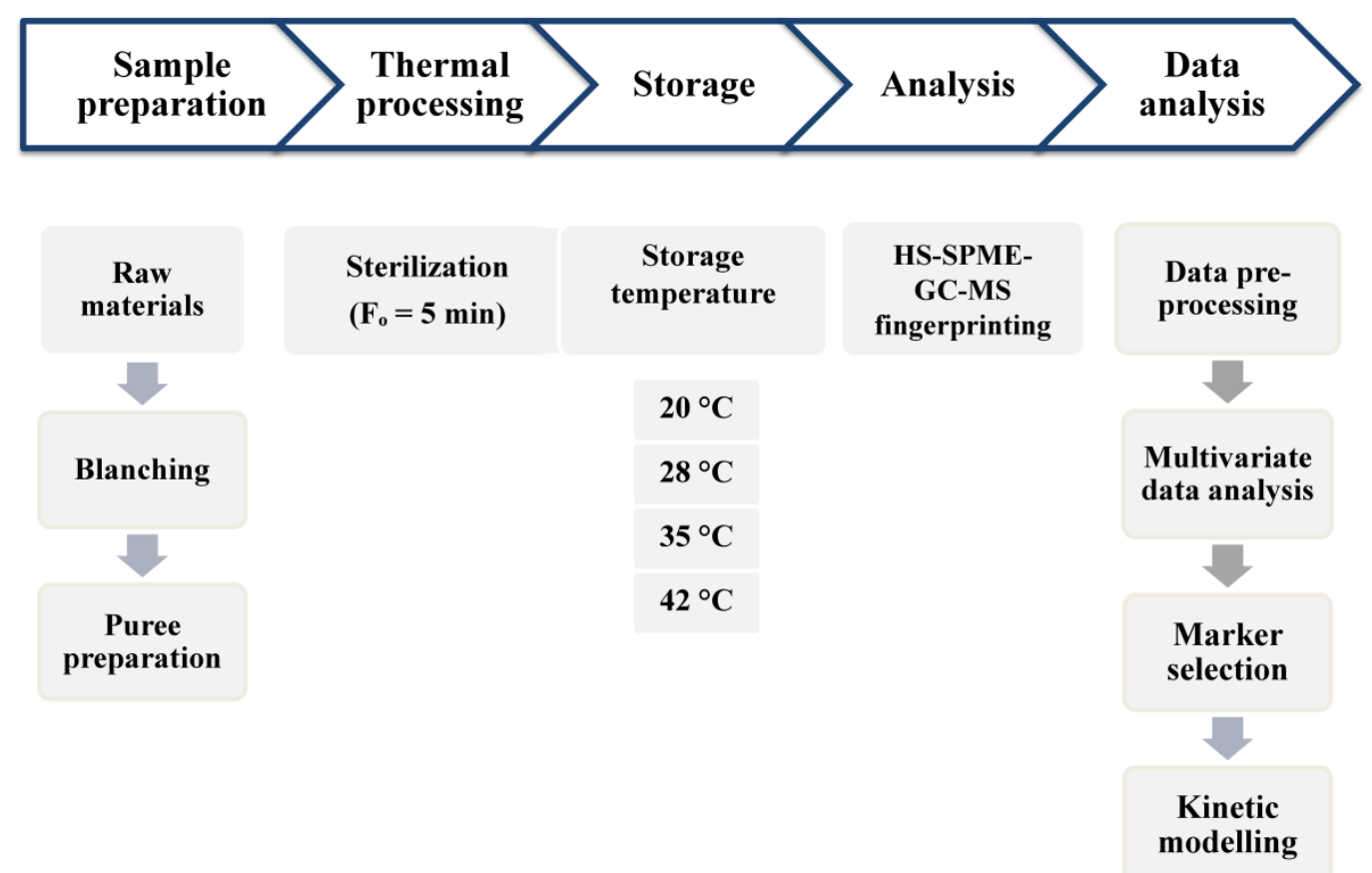

Fig. 1. Experimental set-up for investigating chemical reactions in the headspace fraction of thermally treated carrot puree using a fingerprinting-kinetics strategy.

\subsection{Thermal processing}

The thermal treatment was carried out in a static steriflow pilot retort (Barriquand, Paris, France). An industrially-relevant sterilization value of $F_{121.1^{\circ} \mathrm{C}}^{10^{\circ} \mathrm{C}}\left(F_{0}\right)=5$ min was selected. Glass jars (100 ml volume, $95 \mathrm{~mm}$ height and $45 \mathrm{~mm}$ diameter) were filled with $85 \pm 0.5 \mathrm{~g}$ of carrot puree and closed with metal lids. Temperature profiles in the retort and at the coldest point of the product were recorded using type T thermocouples (Ellab, Hillerød, Denmark). The data logging device provided real-time information of the whole process. For the graphical representation of exemplary time-temperature profiles of the product and environment during treatment, the reader is referred to Kebede et al. (2013). Following completion of the treatments, samples were transferred to ice water to further cool the product down. 


\subsection{Storage}

Sterilized glass jars were stored in incubators, protected from light, at $20{ }^{\circ} \mathrm{C}$ and $28{ }^{\circ} \mathrm{C}$ up to 44 weeks, at $35{ }^{\circ} \mathrm{C}$ up to 26 weeks and at $42{ }^{\circ} \mathrm{C}$ up to 18 weeks. At fixed points in time (11 points per temperature), glass jars were sampled from the incubators. The vegetable puree was aseptically (next to a Bunsen burner) transferred to small-volume (10 $\mathrm{ml})$ polyethylene terephthalate tubes with a polyethylene cap. One gram of sample was taken for microbial analysis. Thereafter, the tubes were frozen in liquid nitrogen, wrapped with aluminium foil and stored in freezer at $-40{ }^{\circ} \mathrm{C}$ until GC-MS analysis.

\subsection{Microbial analysis}

Microbial analysis was performed to verify growth of mesophilic (aerobic) and thermophilic (aerobic and anaerobic) microorganisms. Plate count agar was prepared for aerobic (mesophilic and thermophilic) bacteria, whereas the presence of anaerobic thermophiles were analyzed using reinforced clostridial agar. In the investigated shelf-life time and temperature conditions, the microbial growth was below detection limit.

\subsection{HS-SPME-GC-MS analysis}

Samples were thawed overnight in the cooling room $\left(4{ }^{\circ} \mathrm{C}\right) .2 .5 \mathrm{~g}$ thawed sample and $2.5 \mathrm{ml}$ saturated $\mathrm{NaCl}$ solution were mixed into a $10 \mathrm{ml}$ amber glass vial $(10 \mathrm{ml}$, VWR International, Radnor, PA, USA). The vials were tightly closed using screw-caps with silicon septum seal (GRACE, Columbia, MD, USA), mixed and transferred to the cooling tray of the auto-sampler which was maintained at $10{ }^{\circ} \mathrm{C}$. Headspace fingerprinting was conducted on a gas chromatography (GC) system (7890N, Keysight Technologies, Diegem, Belgium) coupled to a mass selective detector (MSD) (5977N, Keysight Technologies, Diegem, Belgium) and equipped with a combipal autosampler (CTC analytics, Zwingen, Switzerland). Targeting detection of a 
113 wide range of volatiles in a particular food extract, a HS-SPME-GC-MS method of analysis was

114 optimized beforehand (Kebede et al., 2014b). In the selected method, the samples were incubated

115 at $40{ }^{\circ} \mathrm{C}$ during 20 minutes under agitation at $500 \mathrm{rpm}$. Next, extraction of the volatiles was

116 performed using a HS-SPME fiber coated with $30 / 50 \quad \mu \mathrm{m}$

117 divinylbenzene/carboxen/polydimethylsiloxane (DVB/CAR/PDMS) (StableFlex, Supelco,

118 Bellefonte, PA, USA) at $40{ }^{\circ} \mathrm{C}$ during $10 \mathrm{~min}$. The SPME fiber was inserted into the heated (230

$\left.119{ }^{\circ} \mathrm{C}\right) \mathrm{GC}$-injection port for 2 min to desorb the volatile compounds. Prior to extraction, the fibers

120 were conditioned and regenerated according to the manufacturer's guidelines in the conditioning

121 station of the auto-sampler. Injection of the samples to the GC-column was performed in split

122 (1/5) mode. Chromatographic separation was carried out on a HP-5MS capillary column (30 m

$123 \times 0.25 \mathrm{~mm}$ i.d., $0.25 \mu \mathrm{m}$ film thickness, Agilent Technologies J\&W, Santa Clara, CA, USA)

124 coated with 5\%-phenyl-methylpolysiloxane as a stationary phase and helium as a gas phase at a

125 constant flow of $1.2 \mathrm{~mL} / \mathrm{min}$. The GC-oven temperature was programmed from a starting

126 temperature of $40{ }^{\circ} \mathrm{C}$, which was retained for $2 \mathrm{~min}$, to $172{ }^{\circ} \mathrm{C}$ at $4{ }^{\circ} \mathrm{C} / \mathrm{min}$, then ramped to 300

$127{ }^{\circ} \mathrm{C}$ at $30^{\circ} \mathrm{C} / \mathrm{min}$ and kept constant at $300{ }^{\circ} \mathrm{C}$ for 2 min before cooling back to $40{ }^{\circ} \mathrm{C}$. The mass

128 spectra were obtained by electron ionization (EI) mode at $70 \mathrm{eV}$ with a scanning range of 35 to

$129400 \mathrm{~m} / \mathrm{z}$ and a scanning speed of 3.8 scans per second. MS ion source and quadrupole

130 temperatures were $230{ }^{\circ} \mathrm{C}$ and $150{ }^{\circ} \mathrm{C}$, respectively. In this work, to minimize the phenomenon

131 of fiber degradation, a new fiber was used for each storage temperature. Per storage temperature,

132 during analysis, the samples were randomized as a function of storage time. Possible fiber

133 degradation was carefully monitored by analysis of a reference sample (blanched carrot

134 samples), every 10 injections. Per storage time and temperature condition, the analysis was

135 repeated six times. 


\subsection{Data analysis}

137

138

139

140

141

142

143

144

145

146

\subsubsection{Data pre-processing}

As commonly observed in GC-MS analysis, co-eluting compounds were present in the obtained chromatograms. Therefore, all chromatograms were analyzed with automated mass spectral deconvolution and identification system (AMDIS) (Version 2.66, 2008, National Institute of Standards and Technology, Gaithersburg, MD, USA) to extract "pure" component spectra from complex chromatograms. In addition, for proof of identity along with the mass spectral data, AMDIS was used to build a retention index calibration file. The deconvoluted spectra were then analyzed with mass profiler professional (MPP) (Version 12.0, 2012, Keysight Technologies, Diegem, Belgium) for filtering and peak alignment. The MPP yielded a spreadsheet containing peak areas, which was used as an input for the multivariate statistical data analysis.

\subsubsection{Multivariate data analysis and marker selection}

The multivariate data were analyzed with a multivariate data analysis (MVDA) which was carried out in Solo (Version 6.5, 2011, Eigenvector Research, Wenatchee, WA, USA). All data were mean-centered and the variables were weighed by their standard deviation to give them equal variance. In a first step, principal component analysis (PCA) was conducted as an exploratory technique to evaluate each data set and to detect potential outliers. To study the evolution during storage, per storage temperature, partial least squares (PLS) regression was performed, with the volatiles as $X$-variables and the storage time as $Y$-variable. For determining the complexity of the PLS model, the lowest number of latent variables (LVs) that maximally describe the change during storage was used. In PLS, to investigate the change in the volatile fraction as a function of time, bi-plots were plotted. Next, to select volatile compounds clearly changing during storage, variable identification (VID) coefficients were calculated. These values correspond to the correlation coefficient between each original $X$-variable and predicted (by the 
160

161

162

$181 \quad v=\frac{\mathrm{dA}}{\mathrm{d} t}=-k A^{n}$

$182 A=A_{0} \exp (-k t)$ $\left(\mathrm{kJ} / \mathrm{mol}^{*} \mathrm{k}\right)$.

$$
v=\frac{\mathrm{d}}{\mathrm{d} t}=-k A^{n}
$$

selected PLS-model) $Y$-variable. In this work, variables with an absolute VID value higher than 0.70 were considered to be important. These variables were identified and linked to possible reaction pathways. Identification of the compounds was performed by comparing the deconvoluted mass spectrum with the reference mass spectra from both NIST spectral library (NIST08, version 2.0, National Institute of Standards and Technology, Gaithersburg, MD, USA) and WILEY mass spectral data (Wiley2010, version 9, Hoboken, New York, USA). For identification, a threshold match of $90 \%$ was taken into account. For further confirmation, visual inspection of spectral matching between the detected compound and the match from the library as well as comparison of the retention index were performed.

\subsubsection{Kinetic modelling and parameter estimation}

Kinetic modelling was performed based on the rate equation of a degradation reaction. For a detailed discussion on the general principles of kinetic modeling, the reader is referred to the work of van Boekel (2009). The general rate equation of an $n^{\text {th }}$ order degradation reaction is expressed as Equation 1, where $v$ is the reaction rate, $A$ is any property of interest, $n$ is the reaction order and $k$ is the rate constant. Volatiles that were changing during storage could be modelled best by a first-order kinetic model $(n=1)$, where $A_{0}$ is the initial concentration at time $t$ $=0$ (start of storage) and $t$ is the storage time in weeks (Equation 2). The temperature dependency of the reaction rate constant was evaluated using the Arrhenius equation (Equation 3), where $E_{a}$ is the activation energy $(\mathrm{kJ} / \mathrm{mol}), T$ is the storage temperature in kelvin, $k_{\text {ref }}$ is the rate constant $\left(\right.$ weeks $\left.{ }^{-1}\right)$ at reference storage temperature $\left(20{ }^{\circ} \mathrm{C}\right)$ and $R$ is the gas constant 
$183 \quad k=k_{\text {ref }} \exp \left(\frac{E a}{R}\left(\frac{1}{T_{r e f}}-\frac{1}{T}\right)\right)$

184 Model evaluation was performed by examining $\mathrm{R}_{\text {adjusted }}^{2}$ (Equation 4 ) and by visual inspection of

185 the parity plot (estimated values versus measured values) and the scatter plot (residuals versus 186 estimated values). In Equation 4, Where $\mathrm{DF}_{\text {tot }}$ and $\mathrm{DF}_{\text {error }}$ are degree of freedom of total and 187 error, respectively and SS is the sum of squares:

$188 \quad R_{\text {adjusted }}^{2}=1-\frac{\left[\left(\mathrm{DF}_{\mathrm{tot}}-1\right)\left(1-\frac{\mathrm{SS}_{\text {model }}}{\mathrm{SS} \text { total }}\right)\right]}{\mathrm{DF} \text { error }}$

189

190 One-step regression analysis was performed by inserting Equation 3 in Equation 2, using SAS $^{\circledR}$ 191 software (version 9.3, Cary, USA).

\section{RESULTS AND DISCUSSION}

193 As discussed in the introduction, the present work focuses on investigating chemical reactions

194 during shelf-life using an integrated analytical and engineering toolbox: called "fingerprinting-

195 kinetics". In the first step, the potential of fingerprinting was used as fast screening technique for 196 monitoring chemical changes in thermally treated carrot puree as a function of storage.

197 Fingerprinting enabled selection of volatiles of which detected amounts are changing the most 198 during shelf-life. Only these volatiles were identified and further zoomed into (to link them to 199 possible reaction pathways) (section 3.1). In the next step, kinetics was performed to increase 200 insight into the storage temperature dependency of reaction kinetics of volatiles changing as a 201 function of shelf-life. By evaluating the estimated kinetic parameters, the suitability of these 202 volatiles as quality indices (markers) for ASLT was investigated (section 3.2). 


\subsection{Headspace SPME-GC-MS fingerprinting}

204 As discussed in section 2.4, in order to detect a wide range of volatiles, a HS-SPME-GC-MS 205 fingerprinting procedure was optimized. As more than 100 headspace components were detected 206 per storage temperature and compounds are unknowns at the first instance, the procedure can be 207 considered as untargeted for the analyzed particular extract. Fig. 2 depicts an exemplary GC-MS 208 total ion chromatogram of the headspace of thermally treated carrot puree at the start of storage 209 (day 0). As described in section 2.5.1, the complex GC-MS data files were analyzed with a 210 sequence of data preprocessing techniques (i.e., AMDIS and MPP). The MPP obtained a 211 spreadsheet containing peak areas, which was used as an input for the next statistical analysis 212 (MVDA).

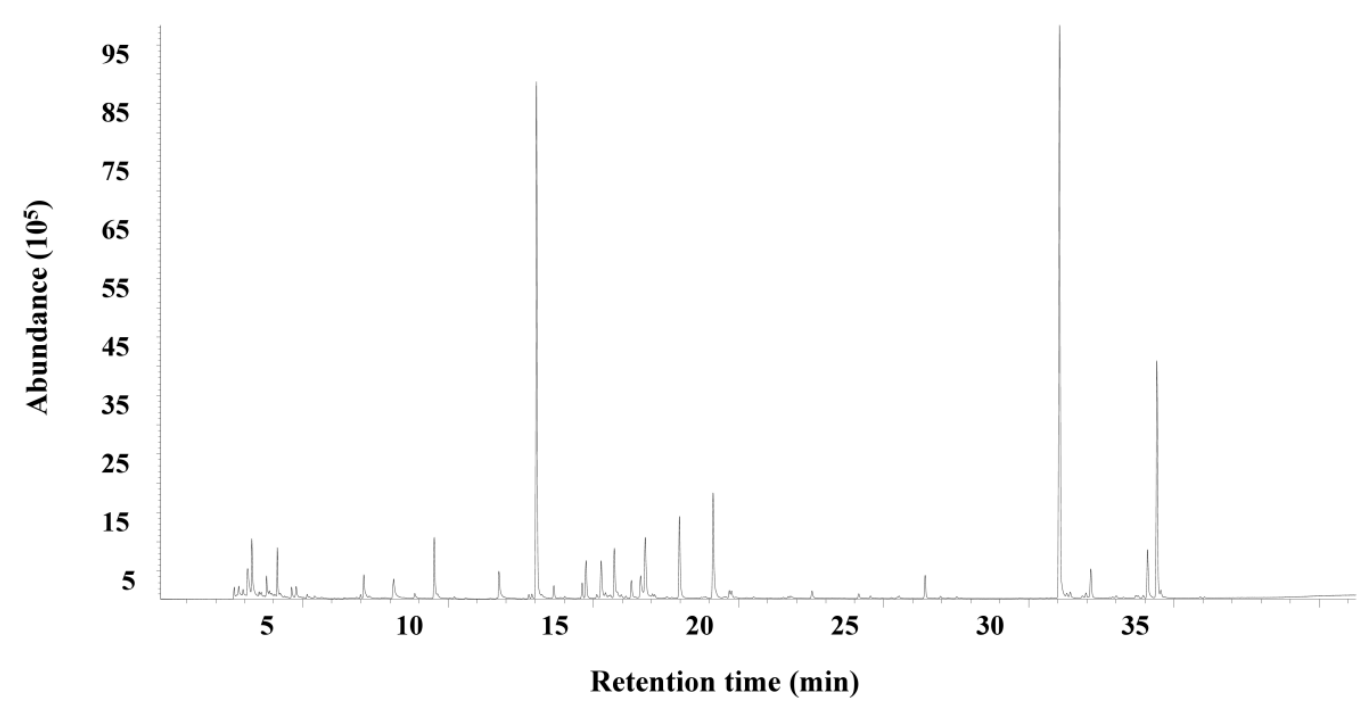

214 Fig. 2. Total ion chromatogram of the headspace of thermally treated carrot puree at the start of storage 215 (day 0), obtained by headspace solid-phase microextraction GC-MS (HS-SPME-GC-MS) fingerprinting. After exploring the data with PCA (data not shown), PLS regression was conducted per storage 218 temperature with the carrot headspace components considered as $X$-variables and storage time as 219 continuous $Y$-variable. For each storage temperature, the first two latent variables (LVs) 

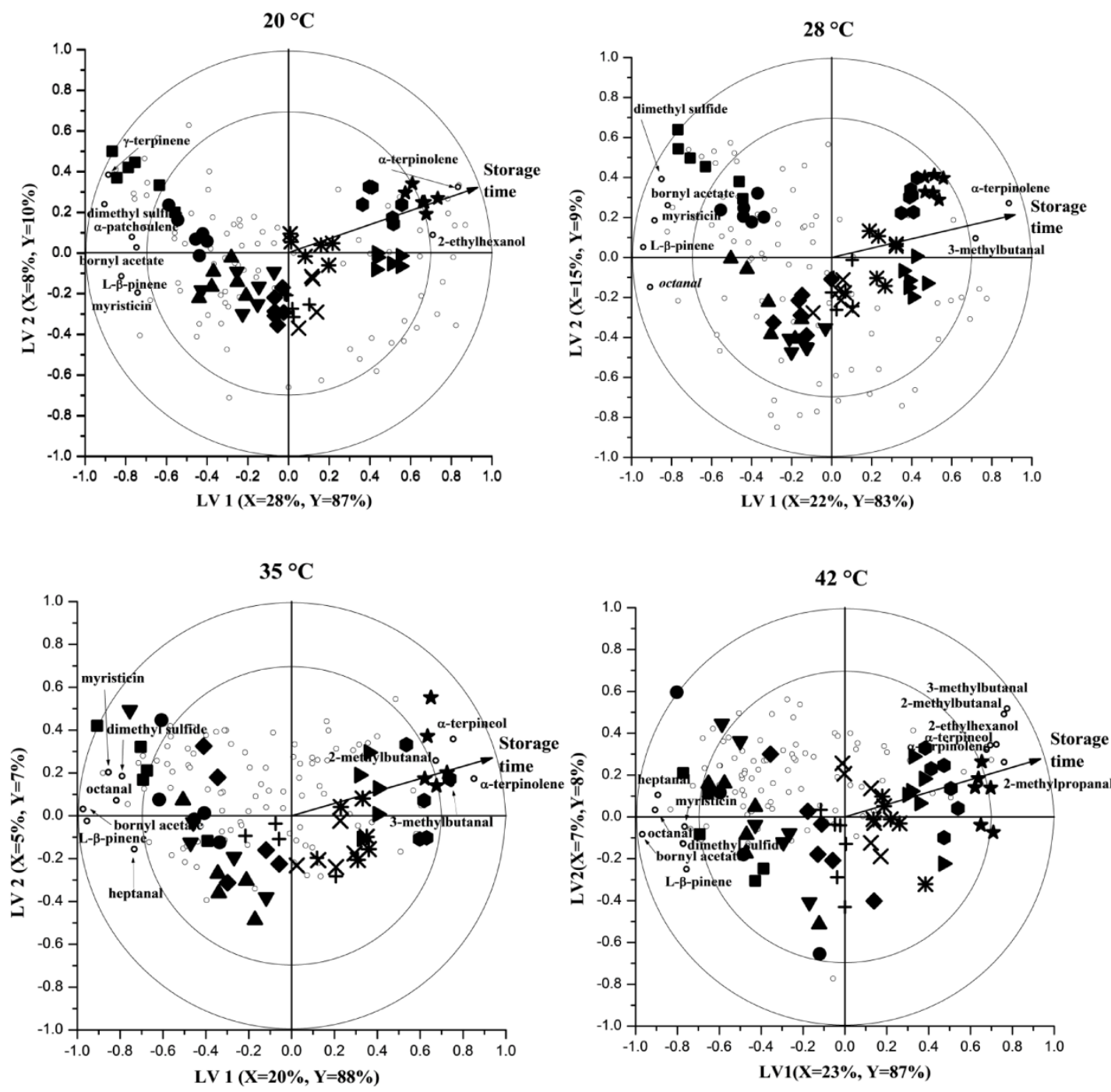

\begin{tabular}{|c|c|c|c|c|c|c|c|c|c|}
\hline & \multicolumn{3}{|c|}{ Storage time (weeks) } & \multirow[b]{2}{*}{$42^{\circ} \mathrm{C}$} & \multicolumn{5}{|c|}{ Storage time (weeks) } \\
\hline & $20^{\circ} \mathrm{C}$ & $28^{\circ} \mathrm{C}$ & $35^{\circ} \mathrm{C}$ & & & $20^{\circ} \mathrm{C}$ & $28^{\circ} \mathrm{C}$ & $35^{\circ} \mathrm{C}$ & $42^{\circ} \mathrm{C}$ \\
\hline - & 0 & 0 & 0 & 0 & $\mathrm{x}$ & 16 & 16 & 10 & 7 \\
\hline - & 1.7 & 1.7 & 1 & 1 & * & 20 & 20 & 14 & 9 \\
\hline$\Delta$ & 4 & 4 & 3 & 1.7 & $\triangleright$ & 28 & 28 & 18 & 11 \\
\hline $\boldsymbol{\nabla}$ & 6 & 6 & 4 & 3 & 0 & 36 & 36 & 22 & 13.6 \\
\hline - & 8 & 8 & 6 & 4 & $\star \star$ & 44 & 44 & 26 & 17.9 \\
\hline$t$ & 12 & 12 & 8 & 5 & & & & & \\
\hline
\end{tabular}

223 Fig. 3. PLS-bi-plots describing the effect of storage on the volatile fraction of thermally treated carrot puree (objects represented by differently shaped symbols) stored at different temperatures, i.e., $20{ }^{\circ} \mathrm{C}, 28$ ${ }^{\circ} \mathrm{C}, 35{ }^{\circ} \mathrm{C}$, and $42{ }^{\circ} \mathrm{C}$. The open circles represent headspace components, of which only components selected by the VID procedure are identified and marked in bold (Table 2). The vectors represent the correlation loading for the $Y$-variable (time). The percentages of the $X$ - and $Y$-variances explained by each latent variable (LV1 and LV2) are indicated on the respective axes. 
Accordingly, for each storage temperature, a multivariate PLS model based on two LVs was

selected. To visualize the multidimensional data structure, bi-plots were constructed per storage

temperature (Fig. 3). A bi-plot is an interesting tool to graphically represent the change in the multidimensional data set, i.e. in the headspace fractions during storage.

233 As can be seen from Figure, the trend of storage time can be clearly observed on the bi-plots.

234 The first clear trend is the horizontal projection of the volatile fractions of the carrot purees from

235 the left of the bi-plot to the right of the bi-plot. This dominant change during storage is described

236 by the first $\mathrm{LV}$, as indicated in the respective axis. The second trend is the u-shaped structure of 237 the Y-variables (volatile fractions). This shows that there is also a variation in the vertical 238 direction in addition to the horizontal direction. This second variation is described by the second 239 LV. The bi-plots also display the relation between headspace compounds and storage time. For 240 instance, compounds that are located in the same direction as the $Y$-vector (the vectors represent 241 the correlation loading for the $Y$-variable (time)) are positively correlated with increasing storage 242 time indicating increase in concentration as a function of storage time, while the ones that are 243 projected in the opposite direction are negatively correlated and decrease with storage time. For 244 samples stored at storage temperatures of $20{ }^{\circ} \mathrm{C}, 28{ }^{\circ} \mathrm{C}$ and $35^{\circ} \mathrm{C}$, most of the components 245 (small open circles, Fig. 3) are projected to the beginning of shelf-life while fewer are positioned 246 close to the end of shelf-life. This seems to suggest that the concentration of most of these 247 compounds have decreased during storage while only few compounds appear to be formed. 248 Nevertheless, more compounds seem to be formed at $42{ }^{\circ} \mathrm{C}$ compared to the other storage 249 temperatures. This phenomenon is further explained in section 3.2. In addition, on the bi-plots, 250 based on the distance of a component from the center of the coordinate, its importance for 251 displaying the changes during storage can be discussed. For instance, if a compound is projected 252 far from the center and located between the two ellipses (inner and outer ellipses represent 
253 correlation coefficients of 70 and $100 \%$, respectively) on the plot, this shows that the

254 concentration of this compound has largely changed as function of storage time.

\section{Table 1}

256 Volatiles significantly changing as a function of shelf-life, per storage temperature $\left(20{ }^{\circ} \mathrm{C}, 28{ }^{\circ} \mathrm{C}, 35^{\circ} \mathrm{C}\right.$

257 and $42{ }^{\circ} \mathrm{C}$ ), selected based on the VID procedure, listed in increasing order of VID coefficient. Positive

258 VID coefficients signify an increase in concentration during storage while negative coefficients denote a

259 decrease. The retention index (RI) and possible chemical group is also listed.

\begin{tabular}{|c|c|c|c|c|}
\hline Storage temperature & VID & Identity & RI & Chemical group \\
\hline \multirow[t]{8}{*}{$20^{\circ} \mathrm{C}$} & -0.85 & dimethyl sulfide & 723 & Sulfur compound \\
\hline & -0.83 & L- $\beta$-pinene & 1055 & Terpene \\
\hline & -0.81 & $\gamma$-terpinene & 1148 & Terpene \\
\hline & -0.76 & myristicin & 1696 & Phenylpropanoids \\
\hline & -0.74 & $\alpha$-patchoulene & 1701 & Terpene \\
\hline & -0.73 & bornyl acetate & 1391 & Terpene \\
\hline & 0.71 & 2-ethylhexanol & 1113 & Alcohol \\
\hline & 0.88 & $\alpha$-terpinolene & 1074 & Terpene \\
\hline \multirow[t]{7}{*}{$28^{\circ} \mathrm{C}$} & -0.91 & octanal & 1083 & Aldehyde (aliphatic) \\
\hline & -0.90 & L- $\beta$-pinene & 1055 & Terpene \\
\hline & -0.81 & myristicin & 1696 & Phenylpropanoids \\
\hline & -0.72 & bornyl acetate & 1391 & Terpene \\
\hline & -0.71 & dimethyl sulfide & 723 & Sulfur compound \\
\hline & 0.72 & 3-methylbutanal & 763 & Aldehyde (Strecker) \\
\hline & 0.93 & $\alpha$-terpinolene & 1074 & Terpene \\
\hline \multirow[t]{10}{*}{$35{ }^{\circ} \mathrm{C}$} & -0.96 & bornyl acetate & 1391 & Terpene \\
\hline & -0.95 & L- $\beta$-pinene & 1055 & Terpene \\
\hline & -0.81 & myristicin & 1696 & Phenylpropanoids \\
\hline & -0.80 & octanal & 1083 & Aldehyde (aliphatic) \\
\hline & -0.76 & dimethyl sulfide & 723 & Sulfur compound \\
\hline & -0.75 & heptanal & 970 & Aldehyde (aliphatic) \\
\hline & 0.70 & 2-methylbutanal & 768 & Aldehyde (Strecker) \\
\hline & 0.76 & 3-methylbutanal & 763 & Aldehyde (Strecker) \\
\hline & 0.80 & $\alpha$-terpineol & 1293 & Terpene-alcohol \\
\hline & 0.87 & $\alpha$-terpinolene & 1075 & Terpene \\
\hline \multirow[t]{12}{*}{$42{ }^{\circ} \mathrm{C}$} & -0.97 & L- $\beta$-pinene & 1056 & Terpene \\
\hline & -0.89 & octanal & 1084 & Aldehyde (aliphatic) \\
\hline & -0.86 & heptanal & 970 & Aldehyde (aliphatic) \\
\hline & -0.79 & dimethyl sulfide & 723 & Sulfur compound \\
\hline & -0.78 & bornyl acetate & 1391 & Terpene \\
\hline & -0.76 & myristicin & 1696 & Phenylpropanoids \\
\hline & 0.72 & $\alpha$-terpinolene & 1075 & Terpene \\
\hline & 0.74 & $\alpha$-terpineol & 1293 & Terpene-alcohol \\
\hline & 0.77 & 2-ethylhexanol & 1113 & Alcohol \\
\hline & 0.79 & 2-methylpropanal & 731 & Aldehyde (Strecker) \\
\hline & 0.83 & 2-methylbutanal & 768 & Aldehyde (Strecker) \\
\hline & 0.85 & 3-methylbutanal & 763 & Aldehyde (Strecker) \\
\hline
\end{tabular}


262 Even though the bi-plot provides graphical representation of the changes, it is not 263 straightforward to rank the relative component importance for the change as a function of storage 264 time. Therefore, to quantitatively rank volatile's importance for the change, VID coefficients 265 were calculated using the selected PLS models (Section 2.6.2). Each volatile was assigned with a 266 value between -1 and +1 , where a positive VID coefficient represents a higher concentration 267 after storage and vice versa. Since the objective was to determine compounds highly changing in 268 concentration, only those with absolute value higher than 0.70 were selected to further zoom into 269 and only those were identified (Table 1; Fig. 3 (bold open circles)). In samples stored at storage 270 temperatures of $20^{\circ} \mathrm{C}, 28^{\circ} \mathrm{C}, 35^{\circ} \mathrm{C}$ and $42{ }^{\circ} \mathrm{C}$, respectively, $8,7,10$ and 12 volatile compounds 271 were selected.

To increase insight into the effect of storage time on quality-related chemical reactions, it was 274 tried to link the significantly changing volatiles to possible reaction pathways. In this work, the 275 selected compounds (Table 1) can be categorized under terpenoids (L- $\beta$-pinene, $\gamma$-terpinene, $\alpha$ 276 patchoulene, $\alpha$-terpinolene, bornyl acetate and $\alpha$-terpineol), phenylpropanoids (myristicin), 277 aldehydes and alcohols (octanal, heptanal, 2-methylpropanal, 2-methylbutanal and 3278 methylbutanal and 2-ethylhexanol) and sulfur-containing (dimethyl sulfide) chemical classes. 279 Prior to thermal sterilization, the carrot cubes were blanched (section 2.1). In addition, from the 280 microbial analysis (section 2.4), no spoilage was detected in the investigated shelf-life time-scale 281 and storage temperatures. Therefore, enzymatic and microbial activities were not expected to 282 have a significant impact on the change in volatiles. In that context, in carrots, the changes of the 283 selected volatiles can be linked to non-enzymatic and non-microbial chemical reactions such as 284 terpenoid and phenylpropanoid degradation, unsaturated fatty acid degradation, Strecker 285 degradation and degradation of sulfur-containing amino acids, respectively. In the following paragraphs, these effects will be discussed step by step in more detail. 
288 On one hand, the concentrations of the majority of terpenoids have decreased during storage, 289 possibly due to degradation into other components. It has been previously described that these 290 compounds are highly thermolabile and sensitive even to low temperature conditions 291 (Heatherbell, Wrolstad, \& Libbey, 1971; Shamaila, Durance, \& Girard, 1996). On the other 292 hand, the amount of two other monoterpenes (e.g. $\alpha$-terpinolene and $\alpha$-terpineol) increased 293 during storage. In literature, formation of $\alpha$-terpineol, which is terpene alcohol, is linked to 294 oxidative processes, in which the tetra-substituted terpenes double bond is attacked (Kjeldsen, 295 Christensen, \& Edelenbos, 2003; Jones, 2008). It can be hypothesized that during storage oxidative conversion of some terpenes to terpene alcohols occurs. With respect to $\alpha$-terpinolene, the literature search didn't reveal a possible explanation for its increased formation during storage at all storage temperatures. Interpreting the selected volatiles and reactions in the context 299 of sensory aspects, the reduction in genuine terpenes might magnify the desired sweet carrot 300 flavor through reducing the harsh or burning-like flavor, which is mostly associated with 301 elevated terpene concentration. Nevertheless, in case of their excessive reduction, the 302 characteristic carrot aroma may be significantly affected (Howard, Braswell, Heymann, Lee, 303 Pike, \& Aselage, 1995; Kjeldsen et al., 2003).

The other group of selected chemical compounds are aliphatic aldehydes: octanal and heptanal, 306 which are mostly linked with autoxidation and/or thermally induced oxidation of unsaturated 307 fatty acids (Heatherbell et al., 1971; Kebede et al., 2013; Kebede et al., 2014a; Kebede et al., 308 2014b). The first stage of these oxidative reactions involves uptake of oxygen in the presence of 309 catalysts, such as transition metals, which is initiated by heat or light. Once highly reactive free 310 radicals are formed, they react in auto-catalytic mode to generate a complex mixture of low 311 molecular weight compounds. Heptanal and octanal have been detected before as volatiles in 
cooked carrots and were reported to increase considerably at high process temperatures (Buttery,

313 Seifert, Guadagni, Black, \& Ling, 1968; Vervoort et al., 2013; Buttery \& Takeoka, 2013). In this

314 work, even though these volatiles are increasingly detected after thermal sterilization, their

315 concentration decreased during storage. It can be hypothesized that after processing, formation

316 of these compounds halted and during storage oxidative breakdown of the aldehydes into other

317 compounds such as aliphatic alcohols (e.g., 2-ethylhexanol) occurred, using the residual oxygen

318 in the system. From sensorial point of view, a certain level of these aliphatic aldehydes and

319 alcohols is generally considered necessary to give characteristic odor and flavor properties to

320 foods, but since many of these aldehydes and alcohols give rise to typical rancid state of flavors,

321 an ideal balance has to be achieved in foods.

323 As can be seen from Table 1, Strecker aldehydes, such as 2-methylpropanal, 2-methylbutanal 324 and 3-methylbutanal, were selected with positive VID values. These volatiles are reaction 325 products of Strecker degradation, one of the side reactions of the Maillard reaction. Although 326 Strecker degradation is a sub-reaction category of the Maillard reaction scheme, it has been 327 described to direct the Maillard reaction towards the aromagenic pathways rather than to 328 chromogenic pathways. In other words, this reaction is of outermost importance in relation to 329 flavor formation (Yaylayan, 2003; van Boekel, 2006; Rizzi, 2008). In our previous studies, 330 comparing the impact of thermal and high pressure high temperature sterilization immediately 331 after processing, Strecker aldehydes were detected at higher levels in a wide range of thermally 332 sterilized vegetable purees (Kebede et al., 2013; Kebede et al., 2014a; Kebede et al., 2014b). 333 However, little information is available regarding the evolution during storage. The present work 334 demonstrates that these compounds further increased as a function of storage. Therefore, it can 335 be hypothesized that the Maillard reaction and its side reactions seem to take place also during 336 storage of thermally sterilized carrot puree. 
338 Dimethyl sulfide is known to be formed from S-methylcysteine, which is a highly thermolabile 339 sulfur containing amino acid derivatives. Previous studies (Vervoort et al., 2013; Kebede et al., 340 2013; Kebede et al., 2014a; Kebede et al., 2014b) reported an increased decomposition of S341 methylmethionine after thermal sterilization leading to the formation of these compounds which 342 are responsible for a typical canned flavor (Bills \& Keenan, 1968; Heatherbell et al., 1971; 343 Kubec, Drhova, \& Velisek, 1998; Araya et al., 2009). In line with the above discussions, in the 344 present work, dimethyl sulfide was detected at higher amount after thermal sterilization. 345 However, the concentrations decreased during storage, which may be attributed to oxidative 346 degradation reactions during storage.

In general it was shown that, per storage temperature, fingerprinting enabled selection of volatile compounds and reactions significantly changing as a function of shelf-life. As discussed before as a second objective in this work, the suitability of these selected volatiles as quality indices

351 (markers) for ASLT was investigated. Based on the data from the fingerprinting as a function of times and temperatures, kinetic modelling of the selected volatiles was performed to investigate their reaction kinetics at different storage temperatures (section 3.2).

\subsection{Reaction kinetics of the changes of headspace components}

Firstly, an appropriate kinetic model was identified. Next, kinetic parameters, such as reaction rate constants and activation energies, were estimated using a non-linear one-step regression analysis (inserting Equation 3 in Equation 2). Of all selected compounds, $\gamma$-terpinene, $\alpha$ patchoulene and 2-ethylhexanol showed a scattering behaviour as a function of storage time, specifically at higher storage temperatures (results not shown). This might be an indication for the presence of non-elementary complex chemical reactions behind the formation of these 
361 products. In addition, the reactions seem to be temperature independent. Hence, these

362 compounds don't seem interesting for ASLT and are not modelled at this stage.

363 Table 2

364 Estimated kinetic parameters based on a one-step first-order kinetic model, Equation $2 \& 3$, $\left(20{ }^{\circ} \mathrm{C}\right.$ as 365 reference temperature) describing changes during storage of discriminant headspace components in 366 thermally treated carrot puree. Samples were stored at $20^{\circ} \mathrm{C}, 28^{\circ} \mathrm{C}, 35^{\circ} \mathrm{C}$ and $42{ }^{\circ} \mathrm{C}$

\begin{tabular}{lccc}
\hline Compound & $\boldsymbol{k}_{\boldsymbol{r e f}}\left(\mathbf{w e e k}^{\mathbf{- 1}}\right)$ & $\mathbf{E a}(\mathbf{k J} / \mathbf{m o l})$ & $\mathbf{r}_{\text {adj }}$ \\
\hline dimethyl sulfide & $0.112 \pm 0.014$ & $14 \pm 6$ & 0.96 \\
bornyl acetate & $0.050 \pm 0.009$ & $15 \pm 9$ & 0.93 \\
myristicin & $0.044 \pm 0.006$ & $37 \pm 7$ & 0.96 \\
$\alpha$-terpinolene & $-0.020 \pm 0.004$ & $49 \pm 7$ & 0.87 \\
L- - -pinene & $0.015 \pm 0.002$ & $72 \pm 5$ & 0.99 \\
$\alpha$-terpineol & $-0.009 \pm 0.005$ & $94 \pm 17$ & 0.76 \\
octanal & $0.015 \pm 0.004$ & $96 \pm 11$ & 0.94 \\
2-methylbutanal & $-0.004 \pm 0.001$ & $96 \pm 10$ & 0.97 \\
heptanal & $0.005 \pm 0.002$ & $108 \pm 16$ & 0.95 \\
2-methylpropanal & $-0.003 \pm 0.002$ & $113 \pm 15$ & 0.94 \\
3-methylbutanal & $-0.002 \pm 0.001$ & $121 \pm 17$ & 0.98 \\
\hline
\end{tabular}

369 The change in the concentration of the rest of the terpenes could be modelled best by means of a

370 first-order empirical kinetic model. The formation of the Strecker aldehydes could be modelled

371 best by means of a zero-order empirical kinetic model. The kinetic parameters and their 372 corresponding $95 \%$ approximate confidence interval are listed in Table 2 . As an example, the 373 changes of L- $\beta$-pinene, 2-methylbutanal and octanal are presented in Fig. 4. In the top section, 374 compound's peak area as a function of time in thermally treated carrot puree stored at $20{ }^{\circ} \mathrm{C}, 28$ $375{ }^{\circ} \mathrm{C}, 35{ }^{\circ} \mathrm{C}$ and $42{ }^{\circ} \mathrm{C}$ is shown. The full lines represent peak area predicted by the kinetic model 376 while the experimental data are represented by the symbols. The model was evaluated, as

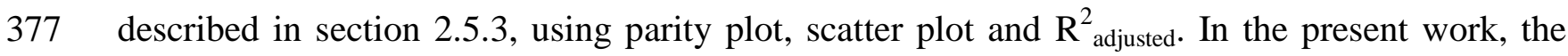
378 observed kinetic parameters are empirical, and thus not necessarily reflecting the actual reaction 379 mechanism. They are, however, a useful tool to obtain insight into the impact of storage on compound changes. 
382 For determining which of these volatiles (from Table 2) could be potential ASLT markers, two criteria were established: (i) the reaction should be temperature-dependent and (ii) there should be an observable change not only at temperature-abuse conditions but also at reference/ambient storage temperature $\left(20^{\circ} \mathrm{C}\right)$. Dimethyl sulfide and bornyl acetate are characterized by very low $E_{a}$-values compared to other compounds, indicating a very low temperature dependency. Hence, 387 given the very small reaction acceleration by increasing storage temperature, these volatiles seem 388 less interesting to be considered as markers for ASLT. For the rest of the compounds, as can be 389 seen from their $E_{a}$-values, increasing the storage temperature effectively increased the rate 390 constants. Nevertheless, for some of them (e.g., heptanal, 2-methylbutanal, 2-methylpropanal 391 and 3-methylbutanal) the formation at $20{ }^{\circ} \mathrm{C}$ proceeds very slow and seem to largely increase at 392 an elevated storage temperature, as indicated by their relatively high activation energies (Table 393 2). As also discussed in section 3.1, these compounds were selected by the VID procedure at all 394 storage temperatures but not at $20{ }^{\circ} \mathrm{C}$, indicating the strong temperature dependency of the 395 reaction kinetics. Therefore, taking into account the second criteria established in this work, care 396 should be taken if and when considering these temperature sensitive volatiles as ASLT markers.

397 Considering both established criteria, myristicin, $\alpha$-terpinolene, L- $\beta$-pinene, $\alpha$-terpineol and 398 octanal seem to be interesting compounds. Their reaction follows Arrhenius kinetics wherein 399 higher storage temperatures lead to the acceleration of the rate of the reaction. In addition, their 400 rate constant is also significant at ambient storage temperature. Therefore, these volatiles 401 compounds can be considered as potential markers for ASLT of thermally sterilized carrot puree.

\section{CONCLUSION}

403 This study clearly showed the power of the integrated fingerprinting-kinetics approach to 404 increase insight into chemical changes during shelf-life of thermally sterilized carrot purees and 
to select markers for ASLT. In a first step, fingerprinting enabled selection of headspace

406 compounds significantly changing during shelf-life. The selected compounds can be categorized

407 into Strecker aldehydes, terpenoids, fatty acid derivatives and sulfur-containing compounds. The

408 concentrations of Strecker aldehydes increased during storage, whereas the majority of terpenes

409 seem to decrease during storage. In the next step, the suitability of these volatiles as markers for

410 accelerated shelf-life testing (ASLT) was investigated. By evaluating the estimated kinetic

411 parameters, myristicin, $\alpha$-terpinolene, L- $\beta$-pinene, $\alpha$-terpineol and octanal were selected as

412 potential markers for ASLT of thermally sterilized carrot puree. In literature, it has been reported

413 that the characteristic aroma and flavor of a carrot is mainly influenced due to terpenes. The fact

414 that the research strategy followed in the present work enabled the selection of terpenes (except

415 octanal) as markers give an indication that ASLT can be used for shelf-life evaluation and

416 estimation of sterilized carrot samples.

417 By evaluating the estimated kinetic parameters, myristicin, $\alpha$-terpinolene, L- $\beta$-pinene, $\alpha$ -

418 terpineol and octanal were selected as potential markers for ASLT of thermally sterilized carrot

419 puree. In general, the applicability of a marker (selected by the fingerprinting-kinetics strategy)

420 to be used for shelf-life estimation can be categorized into three. (i) In cases the marker

421 determines the best before date of the food product and is important for the consumer, the

422 kinetics can be directly used to shelf-life estimation. (ii) If the marker does not directly

423 determine the best before date, but is linked to a reaction causing shelf-life changes, it has a

424 potential to be used as a witness for shelf-life changes and also for shelf-life estimation. (iii) If

425 the latter is not the case, but the kinetics of the marker matches with kinetics of a compound

426 determining the best before date, it has still a potential to be used for ASLT. Based on the

427 obtained results, it is difficult to evaluate to which extent the selected markers affect overall

428 carrot flavor. This was not the aim of the present work, but in the future it is worthwhile to

429 perform a sensory analysis, to understand how the observed changes will be appreciated. In 
430 addition, the impact on different extract of the volatile fraction or on other fraction (e.g. liquid

431 fraction) should be investigated to link the observed changes to other quality attributes, such as

432 vitamins and color.

\section{5. Acknowledgements}

434 This work was financially supported by KU Leuven Research Fund. One of the authors (T.G.) is 435 a postdoctoral researcher funded by the Research Foundation Flanders (FWO) while S.P. was 436 funded by the Agency for Innovation by Science and Technology in Flanders (IWT-Vlaanderen). 437 The authors thank Heidi Roba and Margot De Haes for their laboratory assistance and 438 acknowledge Keysight Technologies, Diegem, Belgium for providing the Mass Profiler 439 professional (MPP) software.

440

441 
Adebooye, O. C., Vijayalakshmi, R., \& Singh, V. (2008). Peroxidase activity, chlorophylls and antioxidant profile of two leaf vegetables (Solanum nigrum L. and Amaranthus cruentus L.) under six pretreatment methods before cooking. International Journal of Food Science and Technology, 43(1), 173-178.

Araya, X. I. T., Smale, N., Zabaras, D., Winley, E., Forde, C., Stewart, C. M., \& Mawson, A. J. (2009). Sensory perception and quality attributes of high pressure processed carrots in comparision to raw, sous-vide and cooked carrots. Innovative Food Science \& Emerging Technologies, 10(1), 420-433.

Bills, D. D. \& Keenan, T. W. (1968). Dimethyl sulfide and its precursor in sweet corn. Journal of Agricultural and Food Chemistry, 16(4), 643-645.

Buttery, R. G., Seifert, R. M., Guadagni, D. G., Black, D. R., \& Ling, L. (1968). Characterization of some volatile constituents of carrots. Journal of Agricultural and Food Chemistry, 16(6), 1009-1015.

Buttery, R. G. \& Takeoka, G. R. (2013). Cooked Carrot Volatiles. AEDA and Odor Activity Comparisons. Identification of Linden Ether as an Important Aroma Component. Journal of Agricultural and Food Chemistry, 61(38), 9063-9066.

Corradini, M. G. \& Peleg, M. (2007). Shelf-life estimation from accelerated storage data. Trends in Food Science \& Technology, 18(1), 37-47.

Grauwet, T., Vervoort, L., Colle, I., Van Loey, A., \& Hendrickx, M. (2014). From fingerprinting to kinetics in evaluating food quality changes. Trends in Biotechnology, 32(3), 125-131.

Heatherbell, D. A., Wrolstad, R. E., \& Libbey, L. M. (1971). Carrot Volatiles .1. Characterization and Effects of Canning and Freeze Drying. Journal of Food Science, 36(2), 219-224.

Hough, G., Garitta, L., \& Gomez, G. (2006). Sensory shelf-life predictions by survival analysis accelerated storage models. Food Quality and Preference, 17(6), 468-473.

Howard, L. R., Braswell, D., Heymann, H., Lee, Y., Pike, L. M., \& Aselage, J. (1995). Sensory Attributes and Instrumental Analysis Relationships for Strained Processed Carrot Flavor. Journal of Food Science, 60(1), 145-148.

Jones, M. J. (2008). Formation of vegetable flavour. In B. Brückner \& S. Grant Wyllie, Fruit and vegetable flavour Recent advances and future prospects (pp. 71-96). Cambridge: Woodhead Publishing Limited.

Kebede, B. T., Grauwet, T., Mutsokoti, L., Palmers, S., Vervoort, L., Hendrickx, M., \& Van Loey, A. (2014a). Comparing the impact of high pressure high temperature and thermal sterilization on the volatile fingerprint of onion, potato, pumpkin and red beet. Food Research International, 56(0), 218-225. 
Kebede, B. T., Grauwet, T., Palmers, S., Vervoort, L., Carle, R., Hendrickx, M., \& Van Loey, A. (2014b). Effect of high pressure high temperature processing on the volatile fraction of differently coloured carrots. Food Chemistry, 153(0), 340-352.

Kebede, B. T., Grauwet, T., Tabilo-Munizaga, G., Palmers, S., Vervoort, L., Hendrickx, M., \& Van Loey, A. (2013). Headspace components that discriminate between thermal and high pressure high temperature treated green vegetables: Identification and linkage to possible process-induced chemical changes. Food Chemistry, 141(3), 1603-1613.

Kjeldsen, F., Christensen, L. P., \& Edelenbos, M. (2003). Changes in volatile compounds of carrots (Daucus carota L.) during refrigerated and frozen storage. Journal of Agricultural and Food Chemistry, 51(18), 5400-5407.

Kubec, R., Drhova, V., \& Velisek, J. (1998). Thermal degradation of S-methylcysteine and its sulfoxide - Important flavor precursors of Brassica and Allium vegetables. Journal of Agricultural and Food Chemistry, 46(10), 4334-4340.

Labuza, T. P. \& Taoukis, P. S. (1990). The relationship between processing and shelf-life. In G. G. Birch, G. Campbell-Plat, \& M. G. Lindley, Foods for the 90's (pp. 73-105). New York: Elsevier Applied Science.

Mizrahi, S. (2000). Accelerated shelf-life tests. In D. Kilcast \& P. Subramaniam, The stability and shelf-life of food (pp. 107-125). Cambridge: Woodhead Publishing Limited and CRC press LLC.

Rizzi, G. P. (2008). The strecker degradation of amino acids: Newer avenues for flavor formation. Food Reviews International, 24(4), 416-435.

Robertson, G. L. (2000). Shelf-Life of Packaged Foods, Its Measurment and Prediction. In G. L. Robertson, Food Packages: Principles and Practices ). New York: CRC press Inc.

Saavedra, J., Cordova, A., Galvez, L., Quezada, C., \& Navarro, R. (2013). Principal Component Analysis as an exploration tool for kinetic modeling of food quality: A case study of a dried apple cluster snack. Journal of Food Engineering, 119(2), 229-235.

Shamaila, M. A. W. E., Durance, T. I. M. O., \& Girard, B. E. N. O. (1996). Water Blanching Effects on Headspace Volatiles and Sensory Attributes of Carrots. Journal of Food Science, 61, 1191-1195.

Sithole, R., McDaniel, M. R., \& Goddik, L. M. (2005). Rate of Maillard Browning in Sweet Whey Powder. Journal of Dairy Science, 88(5), 1636-1645.

van Boekel, M., Fogliano, V., Pellegrini, N., Stanton, C., Scholz, G., Lalljie, S., Somoza, V., Knorr, D., Jasti, P. R., \& Eisenbrand, G. (2010). A review on the beneficial aspects of food processing. Molecular Nutrition \& Food Research, 54(9), 1215-1247.

van Boekel, M. A. J. S. (2006). Formation of flavour compounds in the Maillard reaction. Biotechnology Advances, 24(2), 230-233. 
514 van Boekel, M. A. J. S. (2009). Kinetic Modeling of Reactions in Foods. CRC PRESS515 TAYLOR \& FRANCIS GROUP, 6000 BROKEN SOUND PARKWAY NW, STE 300, BOCA 516 RATON, FL 33487-2742 USA.

517 Vervoort, L., Grauwet, T., Kebede, B. T., Van der Plancken, L., Timmermans, R., Hendrickx, 518 M., \& Van Loey, A. (2012). Headspace fingerprinting as an untargeted approach to compare 519 novel and traditional processing technologies: A case-study on orange juice pasteurisation. Food 520 Chemistry, 134(4), 2303-2312.

521 Vervoort, L., Grauwet, T., Njoroge, D. M., Van der Plancken, I., Matser, A., Hendrickx, M., \& 522 Van Loey, A. (2013). Comparing thermal and high pressure processing of carrots at different 523 processing intensities by headspace fingerprinting. Innovative Food Science \& Emerging 524 Technologies, 18, 31-42.

525 Yaylayan, V. A. (2003). Recent Advances in the Chemistry of Strecker Degradation and 526 Amadori Rearrangement: Implications to Aroma and Color Formation. Food Science and 527 Technology Research, 9(1), 1-6. 